\title{
Differential arthritogenicity of Staphylococcus aureus strains isolated from biological samples
}

\author{
Priscila Maria Colavite-Machado', Larissa Lumi Watanabe Ishikawa', Thaís Graziela Donegá França', \\ Sofia Fernanda Gonçalves Zorzella-Pezavento', Larissa Camargo da Rosa', Fernanda Chiuso-Minicucci', \\ Maria de Lourdes Ribeiro de Souza da Cunha ${ }^{1}$, Gustavo Pompermaier Garlet ${ }^{2}$ and Alexandrina Sartori ${ }^{1 *}$
}

\begin{abstract}
Background: Staphylococcus aureus is the most common agent of septic arthritis that is a severe, rapidly progressive and destructive joint disease. Superantigens produced by S. aureus are considered the major arthritogenic factors. In this study, we compared the arthritogenic potential of five superantigen-producing staphylococcal strains.
\end{abstract}

Methods: Male C57BL/6 mice were intravenously infected with ATCC $19095 \mathrm{SEC}^{+}$, N315 ST5 TSST-1 ${ }^{+}$, S-70 TSST-1 ${ }^{+}$, ATCC 51650 TSST $-1^{+}$and ATCC 13565 SEA $^{+}$strains. Clinical parameters as body weight, arthritis incidence and clinical score were daily evaluated. Joint histopathological analysis and spleen cytokine production were evaluated at the 14th day after infection.

Results: Weight loss was observed in all infected mice. ATCC $19095 \mathrm{SEC}^{+}$, N315 ST5 TSST-1 ${ }^{+}$and S-70 TSST-1 ${ }^{+}$were arthritogenic, being the highest scores observed in ATCC $19095 \mathrm{SEC}^{+}$infected mice. Intermediate and lower clinical scores were observed in N315 ST5 TSST- $1^{+}$and S-70 TSST $-1^{+}$infected mice, respectively. The ATCC 13565 SEA ${ }^{+}$ strain caused death of $85 \%$ of the animals after $48 \mathrm{~h}$. Arthritis triggered by the ATCC $19095 \mathrm{SEC}^{+}$strain was characterized by accentuated synovial hyperplasia, inflammation, pannus formation, cartilage destruction and bone erosion. Similar joint alterations were found in N315 ST5 TSST-1 ${ }^{+}$infected mice, however they were strikingly more discrete. Only minor synovial proliferation and inflammation were triggered by the S-70 TSST- ${ }^{+}$strain. The lowest levels of TNF-a, IL- 6 and IL-17 production in response to $S$. aureus stimulation were found in cultures from mice infected with the less arthritogenic strains (S-70 TSST- $1^{+}$and ATCC 51650 TSST- $1^{+}$). The highest production of IL-17 was detected in mice infected with the most arthritogenic strains (ATCC $19095 \mathrm{SEC}^{+}$and N315 ST5 TSST-1 ${ }^{+}$).

Conclusions: Together these results demonstrated that $\mathrm{S}$. aureus strains, isolated from biological samples, were able to induce a typical septic arthritis in mice. These results also suggest that the variable arthritogenicity of these strains was, at least in part, related to their differential ability to induce IL-17 production.

Keywords: Staphylococcus aureus, Septic arthritis, IL-17

\section{Background}

Staphylococcus aureus is a major cause of bacteremia which frequently leads to infective endocarditis, metastatic abscess formation, toxic shock syndrome, gastroenteritis, pneumonia, osteomyelitis and septic arthritis (SA) [1]. The development of these secondary infections

\footnotetext{
* Correspondence: sartori@ibb.unesp.br

'Department of Microbiology and Immunology, Biosciences Institute, Univ. Estadual Paulista (UNESP), Distrito de Rubião Júnior s/n, 18618-070 Botucatu, São Paulo, Brazil

Full list of author information is available at the end of the article
}

is due to bacterial dissemination from the blood to surrounding tissues and is associated with significantly increased morbidity and mortality [1]. Even though all these secondary infections are severe, SA deserves special attention because it is a rapidly progressive and highly erosive disease of the joints that needs an immediate therapeutical intervention $[2,3]$. The most important risk factor for SA is pre-existing joint disease, especially rheumatoid arthritis (RA) and prosthetic joint surgery [2]. The mortality rate in patients with SA is elevated; around $5-20 \%$ of adults with this disease may die

\section{Biomed Central}


as a consequence of their systemic infection [3]. However, in RA patients that have $S$. aureus infections in more than one joint, the mortality risk increases to $50 \%$ due to the combination of delayed diagnosis, therapeutic immunosuppression, older age and also the polyarticular involvement $[2,3]$.

One of the hallmarks of SA is the massive inflammation that anticipates bone destruction. The infection by $S$. aureus is accompanied by a rapid recruitment of polymorphonuclear granulocytes and activated macrophages that are then followed by $\mathrm{T}$ cells [4]. Although monocytes and macrophages are important to clear bacteria, they also play a pivotal role in the destructive inflammation within the joint [5]. The involvement of proinflammatory cytokines in the pathogenesis of $S$. aureus infection has been reported. This bacteria can induce cytokines such as TNF- $\alpha$, IFN- $\gamma$, IL-1, IL-2, and IL-6 [6,7]. Cytokines released from macrophages as TNF- $\alpha$, IL-1 $\beta$ and IL- 6 have been classically pointed as the major players of the severe inflammation that precedes cartilage and bone destruction in SA [2]. The role of IL-17 in SA is not well established. However, a possible deleterious role is highly supported by many reports in the areas of rheumatoid arthritis and osteoarthritis [8]. IL-17A appears to play a key role in host defense against local $S$. aureus infections by inducing the production of neutrophil-mobilizing chemokines, colony-stimulating factors, and cytokines [9].

$S$. aureus strains can produce a number of different components that may contribute to virulence and arthritogenicity, including surface-associated adhesins, capsular polysaccharides, clumping factor A, exoenzymes, and exotoxins [10-12]. Some of the toxins produced by $S$. aureus are called superantigens (SAgs) because they are endowed with the ability to activate various $\mathrm{T}$ cell clones, independently of their specificity. These SAgs mediate $\mathrm{T}$ cell activation in a very distinctive way from conventional antigens. These molecules are able to simultaneously bind to class II molecules, on antigen presenting cells, and to a large $\mathrm{T}$ cell population comprising all clones that share certain variable regions in the TCR V $\beta$ chain [13]. They cause fever, hypotension and other acute toxic-shock-like symptoms by inducing the release of pro-inflammatory cytokines, such as IFN- $\gamma$, TNF- $\alpha$, IL-1 and IL-12 $[14,15]$.

Several studies indicate that experimental staphylococcal arthritis in mice is the best model to study SA because of the striking resemblances between the murine and human immune systems [16,17]. The characteristics of the murine model closely mirror changes seen in human SA, especially with regard to the high frequency and severity of periarticular bone erosivity [4]. LS-1 strain is able to produce TSST-1 and is the most employed $S$. aureus strain to trigger experimental SA
$[5,16,17]$. The main objective of this work was to compare the arthritogenic potential of various SAg-producing staphylococci. Disease incidence, clinical scores, histopathological alterations and cytokine production were the criteria used to characterize arthritis severity.

\section{Methods}

\section{Experimental design}

Mice were infected with different $S$. aureus strains and were daily evaluated by a clinical follow-up that included weight determination, disease incidence and individual clinical scores. Fourteen days after infection they were euthanized and submitted to histopathological and immunological analysis. Cellular immunity was checked considering cytokine production by spleen cells stimulated with $S$. aureus and Concanavalin A (ConA). Noninfected animals were included as a control group. Each group contained 5-9 animals.

\section{Animals}

Male C57BL/6 mice (8-10 weeks old) were purchased from PUSP-RP (USP, São Paulo, SP, Brazil). The animals were fed with sterilized food and water ad libitum and were manipulated in accordance with the ethical guidelines adopted by the Brazilian College of Animal Experimentation. All experimental protocols were approved by the local ethics committee for animal experimentation (CEEA), Medical School, Univ. Estadual Paulista (protocol number 291).

\section{S. aureus strains and culture conditions}

The following SAg producer strains were used: ATCC 19095 SEC $^{+}$, N315 ST5 TSST-1 ${ }^{+}$, S-70 TSST-1 ${ }^{+}$, ATCC $51650 \mathrm{TSST}^{-}{ }^{+}$, and ATCC $13565 \mathrm{SEA}^{+}$. Information related to original isolation and investigations done with these strains is depicted in Table 1 . Before each experiment, bacteria were cultured in blood agar plates (Merck) for $24 \mathrm{~h}$ at $37^{\circ} \mathrm{C}$ in order to confirm their purity and to determine their morphology and specific color. Isolated colonies were inoculated in brain heart broth (BHI, Merck) and incubated in $37^{\circ} \mathrm{C}$ for $24 \mathrm{~h}$. Bacteria were collected by centrifugation, washed three times and resuspended in cold sterile saline, as described by França et al., (2009) [18]. The bacterial suspensions were prepared according to the McFarland nephelometer $n^{\circ} 0.5$. The exact amount of live bacterial cells was determined by further enumeration of the number of colony forming units (CFU) on agar plates.

\section{Arthritis induction}

Disease was induced according to the methodology described by Bremell et al. (1991), slightly modified [17]. The infection was performed through the retro-orbital 
Table 1 S. aureus strains: origin and infection doses

\begin{tabular}{|c|c|c|c|}
\hline Strains & Source & Inoculum & Reference \\
\hline ATCC 19095 SEC $^{+}$ & Leg abscess of a patient (Albert Merritt Billings Hospital, University of Chicago, 1933) & $2.2 \times 10^{7}$ & [19] \\
\hline N315 ST5 TSST-1 ${ }^{+}$ & Pharyngeal smear of a japanese patient, 1982 & $9.4 \times 10^{7}$ & [20] \\
\hline S-70 TSST- $1^{+}$ & Secretion of a newborn patient (Botucatu, Brazil, 2007) & $7.9 \times 10^{8}$ & [21] \\
\hline 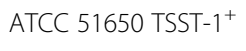 & Wound of a patient with nonmenstrual toxic shock syndrome, Vancouver, British Columbia, Canada & $7.8 \times 10^{11}$ & [22] \\
\hline ATCC 13565 SEA $^{+}$ & Food poisoning (Vancouver, Wash, 1940) & $1.3 \times 10^{7}$ & [23] \\
\hline
\end{tabular}

route instead of the caudal vein as originally described. Each animal was infected with $0.2 \mathrm{~mL}$ of a S. aureus suspension made in physiological saline and control mice were injected with $0.2 \mathrm{~mL}$ of this diluent. The exact amount of bacteria injected in each group is indicated in Table 1.

\section{Clinical evaluation}

Arthritis was defined as a visible joint erythema and/or swelling of at least one joint. Mice were individually analyzed and joints were inspected every day. The number of arthritic limbs per animal was registered. Arthritis intensity was recorded as described by Abdelnour et al. (1993) [24]. Briefly, the arthritic index (clinical score) was carried out by using a system where macroscopic inspection yielded a score of $0-3$ points for each limb ( 1 point $=$ mild swelling and $/$ or erythema; 2 points $=$ moderate swelling and erythema; 3 points $=$ marked swelling and erythema). The clinical score of each group was determined by dividing the total score (sum of the scores of all animals of each group) by the total number of animals in each group.

\section{Histopathological examination}

Joint histopathological examination was done 14 days after infection. After fixation by $10 \%$ formaldehyde, the joints were decalcified for 8 weeks in a solution with $18 \%$ of ethylenediamine tetraacetic acid. After confirmation that joints were decalcified by a radiographical procedure, they were washed, dehydrated, and embedded in paraffin. Serial sections with $5 \mu \mathrm{m}$ thickness were cut and stained with haematoxylin and eosin. The sections were semi-quantitatively evaluated in relation to the presence of inflammatory infiltrates, synovial membrane hyperplasia, pannus formation, cartilage destruction and bone erosion.

\section{Cytokine quantification}

Control and infected animals were euthanized 14 days after infection. Spleen cells were collected and adjusted to $5 \times 10^{6}$ cells $/ \mathrm{mL}$. Cells were cultured in complete RPMI medium (RPMI supplemented with $5 \%$ of fetal calf serum, $20 \mathrm{mM}$ glutamine and $40 \mathrm{IU} / \mathrm{mL}$ of gentamicin). Cultures were stimulated with a standardized preparation of S. aureus (Pansorbin from Calbiochemical) or
ConA (Sigma-Aldrich). Pansorbin is a suspension of heat-killed and formalin-hardened S. aureus Cowan I cells and was used at a final dilution of 1:2500 (v:v); ConA was used at a final concentration of $10 \mu \mathrm{g} / \mathrm{mL}$ in the cell culture. Cytokine levels were evaluated $48 \mathrm{~h}$ later by enzyme-linked immunosorbent assay (ELISA) in culture supernatants using IFN- $\gamma$ BD OptEIA Sets (Becton Dickinson) and IL-6, IL-17 and TNF- $\alpha$ Duosets (R\&D Systems, Minneapolis, MN, USA). The assays were performed according to the manufacturer's instruction.

\section{Statistical analysis}

Data were expressed as mean \pm SE. Comparisons between infected groups were made by Student's test or one way ANOVA with Tukey test for parameters with normal distribution. Significance level was $\mathrm{p}<0.05$. Statistical analysis was accomplished with SigmaStat for Windows v 3.5 (Systat Software Inc).

\section{Results}

\section{Weight loss}

Body weight was daily checked. The percentage of weight variation after 3,7 and 14 days of infection is illustrated in Figure 1. As can be observed, in spite of some variation, infection with all strains usually determined a significant weight loss.

\section{Arthritis incidence}

The kinetics of arthritis development was very similar in the groups infected with ATCC $19095 \mathrm{SEC}^{+}$and N315 ST5 TSST $-1_{\text {. }}^{+}$Both groups already presented clinical disease signs (edema and/or erythema) 24 hours after infection. They also reached around $80 \%$ of disease incidence. The S-70 ${\text { TSST }-1^{+}}^{+}$infected group presented delayed clinical manifestation that occurred only at the 5th day of infection. A $100 \%$ disease incidence was observed in this group at the 10th day of infection. The ATCC 51650 TSST $-1^{+}$strain did not trigger any sign of arthritis during the planned experimental period. These results are shown in Figure 2a. The ATCC $13565 \mathrm{SEA}^{+}$strain caused death of $85 \%$ of the animals in $48 \mathrm{~h}$ (not shown).

\section{Clinical scores}

Clinical scores are shown in Figure 2b. The highest clinical scores were observed in the group infected with the 


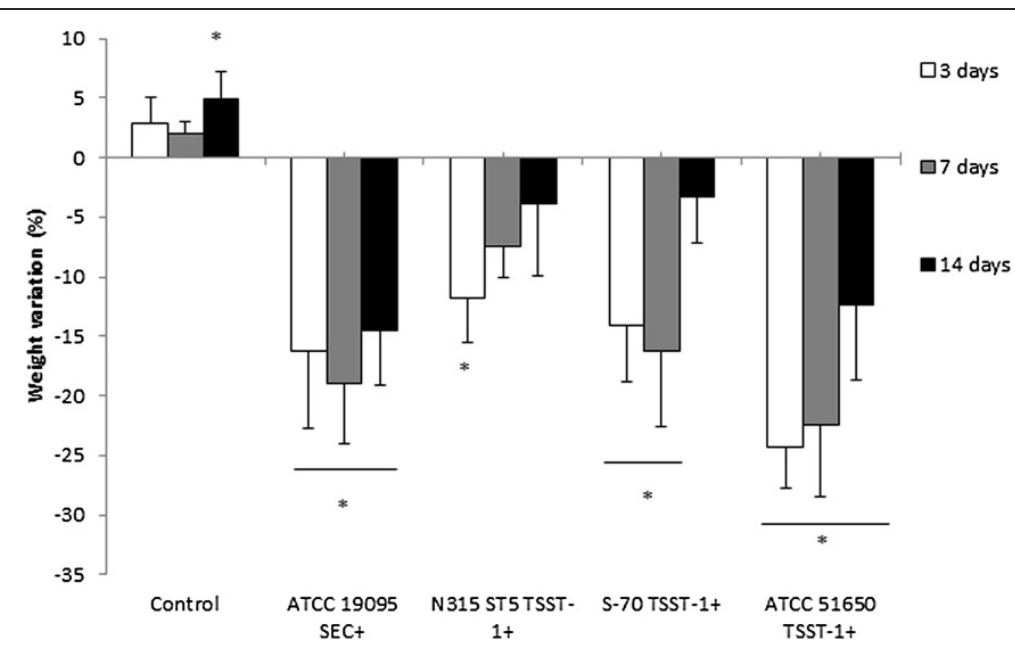

Figure 1 Weight variation in C57BL/6 mice infected with S. aureus strains. Animals were infected with ATCC $19095 \mathrm{SEC}^{+}$, N315 ST5 TSST-1 ${ }^{+}$, S-70 TSST-1 ${ }^{+}$or ATCC 51650 TSST-1 ${ }^{+}$. Weight variation at 3, 7 and 14 days after infection is illustrated. Data is presented by mean \pm SE of 5-9 mice. *represents the difference between initial weight and the weight in each time interval. $p<0.05$.
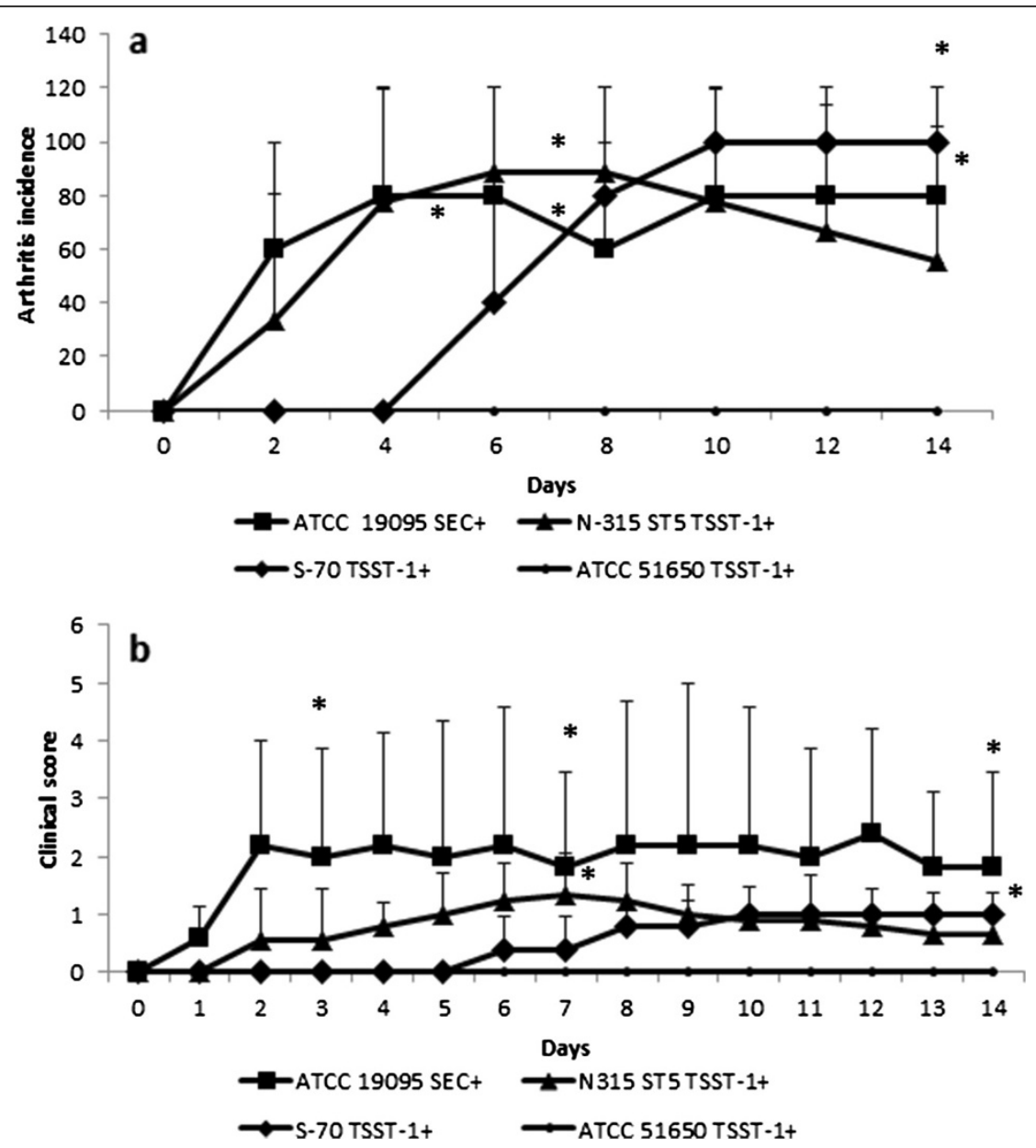

Figure 2 Arthritis development in C57BL/6 mice infected with S. aureus strains. Animals were infected with ATCC 19095 SEC ${ }^{+}$, N315 ST5 $\mathrm{TSST}_{-1}{ }^{+}, \mathrm{S}-70 \mathrm{TSST}-1^{+}$or ATCC $51650 \mathrm{TSST}^{-1}{ }^{+}$. (a) Arthritis incidence and (b) clinical score were daily evaluated. Data is presented by mean \pm SE of 5-9 mice. * represents the difference with the group infected with the non arthritogenic ATCC 51560 TSST- ${ }^{+}$strain. $p<0.05$. 
ATCC $19095 \mathrm{SEC}^{+}$strain. In spite of some variation, these high scores were maintained until the end of the experimental period. Even though hind paws and forepaws were affected, the hind paws presented higher scores (not shown). Lower clinical scores were observed in N315 ST5 TSST- $1^{+}$and S-70 TSST- $1^{+}$infected mice. The ATCC 51650 TSST $-1^{+}$did not provoke any clinical sign of arthritis. The ATCC $13565 \mathrm{SEA}^{+}$strain was lethal to $85 \%$ of the animals, but the remaining $15 \%$ did not develop any clinical sign of arthritis.

\section{Histopathological analysis}

The normal histological pattern of mice joints is shown in Figures 3a and 3a'. A very thin synovial layer, a marked joint cavity, an intact cartilage and a preserved bone structure can be observed. After 14 days of infection the histological aspect of swollen joints from forepaws was very distinct in the three experimentally infected groups. Mice infected with the ATCC 19095 $\mathrm{SEC}^{+}$strain presented a severe arthritis characterized by a marked proliferation of synovial tissue and a huge inflammatory process. The inflammatory infiltrate was mainly localized inside the synovial cavity but it also penetrated into the cartilage and the bone. A striking pannus formation occupied almost the entire joint cavity. Cartilage and bone erosion were also observed in many areas (Figures 3b and 3b'). Synovial hyperplasia, inflammation, pannus formation and cartilage and bone erosion were also detected in mice infected with the N315 ST5 TSST- $^{+}$strain but in lesser extension (Figures $3 c$ and 3c'). As shown in Figures 3d and 3d', only minor degrees of synovial proliferation and inflammation were detected in the joints of mice infected with the S-70 ${\text { TSST }-1^{+}}^{+}$strain. Arthritis in the hind paws of ATCC 19095 SEC $^{+}$infected animals was even more severe than in the forepaws. In this case, the synovial space was entirely occupied by inflammatory infiltrates and bone tissue was severely eroded (Figures $4 b$ and 4b'). Various cell types as polymorphonuclear cells, mononuclear cells and fibroblast-like cells were observed. Granuloma-like structures were also visualized in these joints. Histopathological changes were not detected in the hind paws of animals infected with N315 ST5 TSST$1^{+}$or S-70 TSST $-1^{+}$S. aureus strains as can be observed in Figures $4 \mathrm{c}$ and $4 \mathrm{~d}$.

\section{Cytokine production}

The cellular immune response was evaluated by the production of cytokines by spleen cells stimulated with fixed $S$. aureus Cowan strain I or ConA. High levels of IFN- $\gamma$ were produced by cells stimulated with $S$. aureus. Even though these levels were generally similar to the ones produced by cells from control (non-infected) mice, the ATCC 19095 SEC $^{+}$and S-70 TSST-1 ${ }^{+}$infected groups produced significantly higher amounts than the ATCC

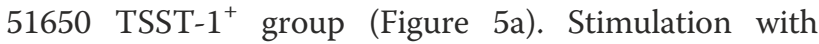
ConA triggered elevated IFN- $\gamma$ production by all experimental groups including, as expected, the control group (Figure 5a). All groups produced TNF- $\alpha$ when the cells were stimulated with $S$. aureus, being the levels found in the control, ATCC 19095 SEC $^{+}$and N315 ST5 TSST-1 ${ }^{+}$ similarly elevated. The levels of this cytokine were, however, significantly lower in the groups S-70 $\mathrm{TSST}_{-1}{ }^{+}$and ATCC 51650 TSST $-1^{+}$(Figure 5c). Production of IL-6 induced by $S$. aureus was significantly lower in the group infected with $S$. aureus S-70 TSST-1 $1^{+}$(Figure 5b). A very similar profile was observed when the cultures were stimulated with ConA, i.e., significantly lower levels of IL-6 were produced by mice infected with S-70 TSST- $1^{+}$ and ATCC $51650{\text { TSST }-1^{+}}^{+}$strains in comparison to

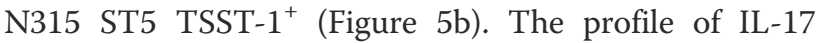
production was similar in the cultures stimulated with $S$. aureus and ConA, being its levels higher in cultures from N315 ST5 TSST-1 ${ }^{+}$and ATCC 19095 SEC $^{+}$groups as can be observed in Figure $5 \mathrm{~d}$. The most striking differences were, however, observed in the cultures stimulated with $S$. aureus. In this case, IL-17 levels were significantly higher in the groups N315 ST5 TSST- $1^{+}$ and ATCC $19095 \mathrm{SEC}^{+}$in comparison to all other groups (Figure $5 \mathrm{~d}$ ).

\section{Discussion}

Septic arthritis is an infectious disease that affects the joints. Due to its fast evolution, even with prompt therapy, it can cause irreversible joint damage and even death [2]. $S$. aureus is the most common causative agent of this disease and it has been believed that SAg-production plays a pivotal role in arthritogenicity $[2,15]$. The main goal of this work was to compare the arthritogenic potential of SAgproducing $S$. aureus strains considering disease incidence, clinical score and histopathological alterations. Cytokine production was also evaluated to get some insight into possible differences among the various strains. An initial screening process was performed to evaluate susceptibility of $\mathrm{C} 57 \mathrm{BL} / 6$ and BALB/c mice to develop septic arthritis after $S$. aureus infection. Gender effect was also checked. This preliminary evaluation, that was done only with the ATCC $19095 \mathrm{SEC}^{+}$strain, indicated that male and female, from both mice strains, lost a significant percentage of weight in the first days of infection. We believe that all animals were similarly infected because weight loss is accepted as a parameter to indicate effective infection [25]. However, only C57BL/6 male mice developed arthritis. This resistance of $\mathrm{BALB} / \mathrm{c}$ mice to develop experimental arthritis was already described by Bremmel et al. (1991) [17]. Our results were, however, distinct from the more recently published data of Henningsson et al. (2010) [26]. These authors described a high incidence of arthritis in 

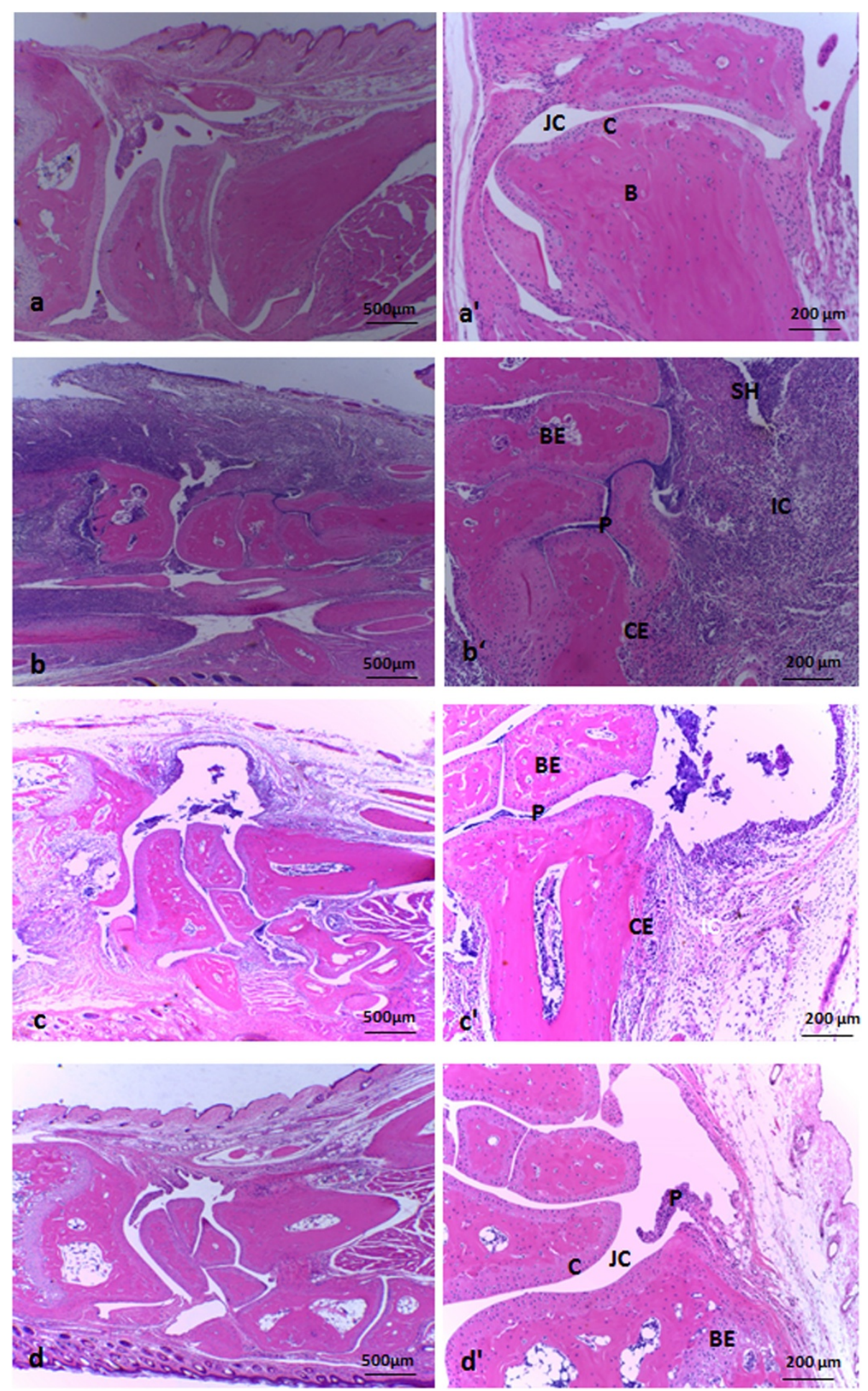

Figure 3 Histopathological analysis in forepaw joints from C57L/6 mice infected with S. aureus strains. (a, a') non-infected control. (b, b')

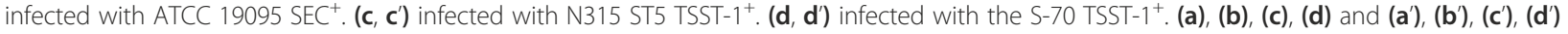
represent histopathological micrographics with $4 \mathrm{x}$ and 10x magnification, respectively. JC, joint cavity; C, cartilage; $\mathrm{B}$, bone; $\mathrm{SH}$, synovial hyperplasia; BE, bone erosion; CE cartilage erosion; P, pannus formation and IC, inflammatory cells. Panel is representative of 5-9 animals/group. 

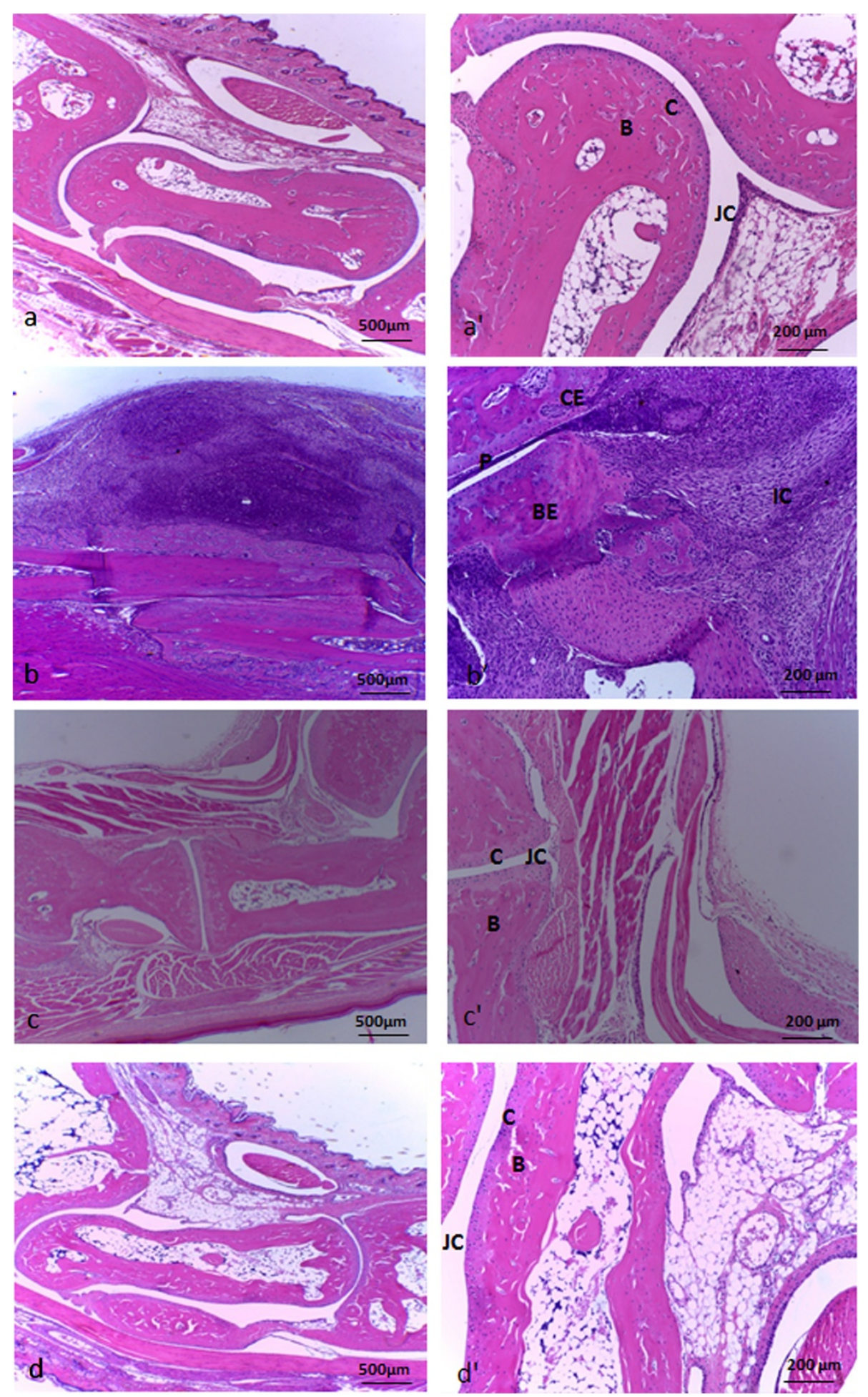

Figure 4 Histopathological analysis in hind paw joints from C57L/6 mice infected with S. aureus strains. (a, a') non-infected control. (b, b')

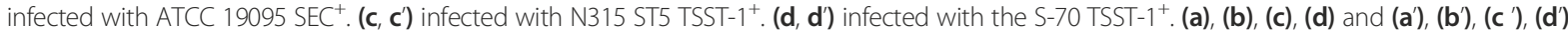
represent histopathological micrographics with $4 x$ and 10x magnification, respectively. JC, joint cavity; C, cartilage; $B$, bone; $B E$, bone erosion; CE cartilage erosion; P, pannus formation and IC, inflammatory cells. Panel is representative of 5-9 animals/group. 
both male and female C57BL/6 mice infected with the LS-1 TSST $-1^{+}$S. aureus strain. This variable susceptibility could be related, at least partially, to $S$. aureus strain particularities. C57BL/6 male mice were, therefore, chosen to compare the arthritogenic potential of the different $S$. aureus strains. These animals were then infected by the retroorbital plexus and body weight and clinical scores were daily checked until the 14th day when animals were euthanized for histopathological and immunological evaluations. The fact that inoculation of all 5 strains triggered significant weight loss, suggests successful experimental infections as has been proposed by other authors [25].

Arthritis incidence and clinical scores varied among S. aureus strains. ATCC $19095 \mathrm{SEC}^{+}$and N315 ST5 $\mathrm{TSST}_{-} 1^{+}$were the most arthritogenic ones. They triggered earlier symptoms and the highest levels of incidence. The ATCC $19095 \mathrm{SEC}^{+}$strain was associated with the highest clinical scores. The S-70 TSST- ${ }^{+}$ infected group presented a delayed clinical manifestation and lower clinical scores whereas the ATCC 51650
TSST $-1^{+}$strain did not cause any sign of arthritis during 14 days. The ATCC 13565 SEA $^{+}$provoked death of $85 \%$ of the animals after $48 \mathrm{~h}$ (not shown). If these differential outcomes in mice can be translated to human SA is a subject that needs further investigation. Many bacterial components are thought to contribute to arthritogenicity [10-12]. The delayed appearance of arthritis in S-70 TSST $-1^{+}$infected mice could mean, for example, that this strain is endowed with a smaller number of arthritogenic factors than the other ones. In this scenario, we could think that this strain is being better controlled by the innate immunity. Alternatively, some strains, as seems to be the case of S-70 TSST- $1^{+}$, could be less inflammatory. The level of pro-inflammatory cytokines showed by our results indicates that this less arthritogenic strain induced lower levels of TNF- $\alpha$, IL- 6 and IL-17 than the two more arthritogenic ones.

No direct relationship was found between arthritogenicity and bacterial inoculum. By comparing bacterial inoculum with the degree of arthritis severity, we can conclude that

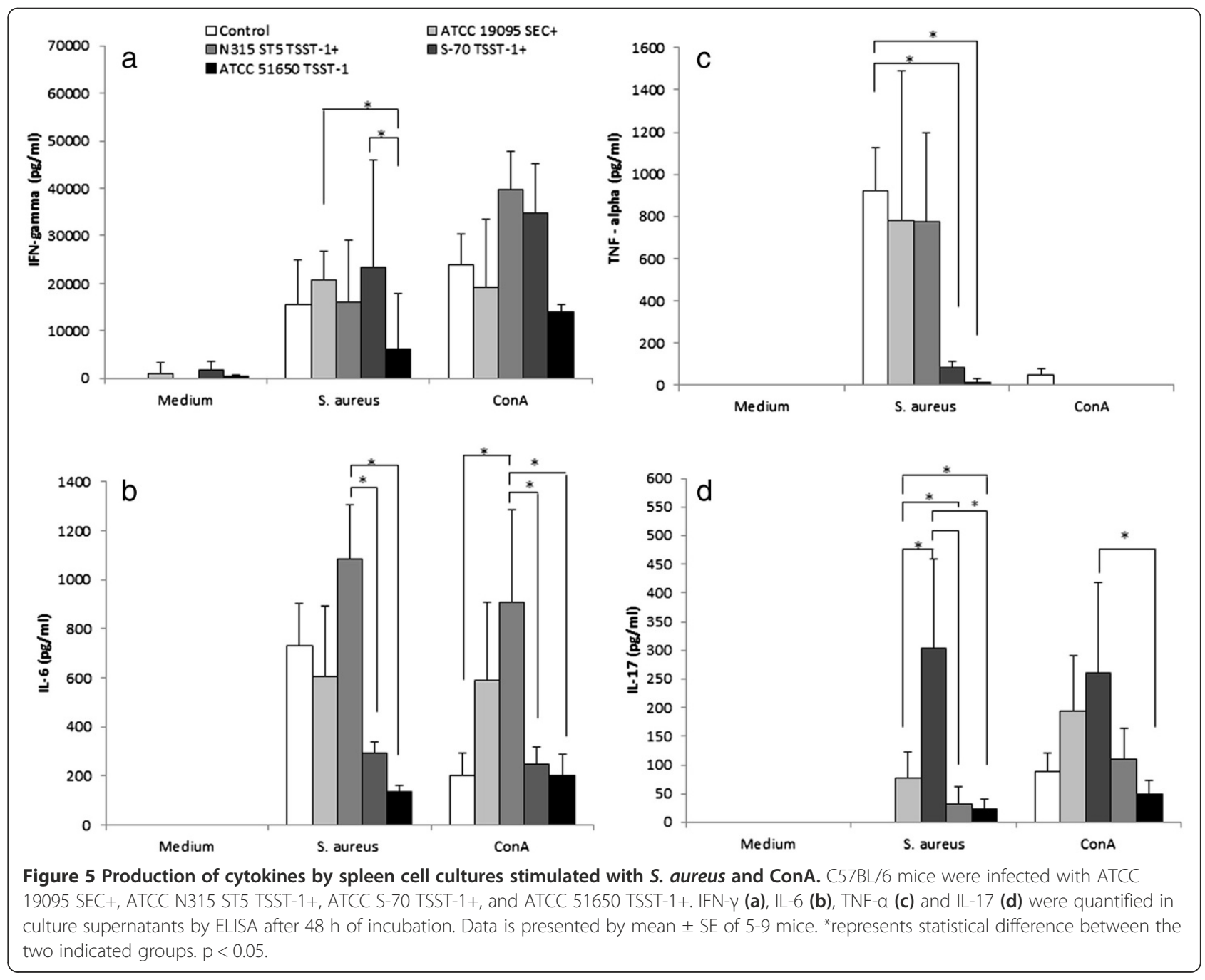


the differential arthritis severity was not associated with distinct bacterial concentrations. We cannot rule out, however, the possibility that these strains present distinct potential to colonize the joints. Unexpectedly, even though the bacterial suspensions from the 5 strains were prepared by the same methodology, they really contained distinct number of viable bacteria. This subject was not further evaluated but it suggests that these preparations could have distinct proportions of dead and alive bacteria. Concerning this differential arthritogenicity among $S$. aureus strains we would like to highlight two aspects. This is the first demonstration that these strains, that were originally isolated from biological samples, can cause septic arthritis in mice. In addition, these results indicate that superantigenicity was not enough to elicit arthritis. The ATCC 51650 TSST- $1^{+}$strain, for example, was not able to induce septic arthritis, even though it was used in the adequate range of bacterial concentration [16]. This finding is in accordance with the postulate that induction of arthritis by $S$. aureus infection is likely elicited by the concerted action of multiple events as activation of T lymphocytes by SAgs, exposure to peptidoglycans/capsular polysaccharides, presence of surface-associated adhesins, clumping factor A and also free bacterial DNA [2,10-12]. Even though not clearly demonstrated in the literature, the ability of $S$. aureus to form biofilms with joint components is being suggested as a possible arthritogenic element $[27,28]$. In this sense, it would be very enlightening to compare the ability of these $S$. aureus strains to form biofilms.

The histopathological analysis of the joints revealed the presence of synovial proliferation, pannus formation and inflammatory infiltrates in the joint cavity. These findings are similar to the most consensual features of SA caused by $S$. aureus [29]. Cartilage and bone erosion were also present, mainly in joints from animals infected with the ATCC $19095 \mathrm{SEC}^{+}$strain. This evolution from the inflammatory process to cartilage and bone erosion is very relevant because it mimicries the human situation during SA by $S$. aureus. In this case, $25-50 \%$ of the patients progress to bone destruction and irreversible loss of joint function [30-32].

TNF- $\alpha$, IL- 6 and IL-17 are being described as some of the most relevant mediators in SA immunopathogenesis $[26,33]$. To investigate the possible contribution of cytokines to these histopathological alterations, the production of TNF- $\alpha$, IFN- $\gamma$, IL- 6 and IL-17 was simultaneously quantified in spleen cultures stimulated with $S$. aureus or ConA. Even though all of them can be released during the initial innate immunity, IFN- $\gamma$ and IL-17 can also be produced during specific immunity by effector Th1 and Th17 cells, respectively. Interestingly, the lowest levels of TNF- $\alpha$, IL-6 and IL-17 were found in cultures from mice that presented the lowest clinical scores. In addition, differently from IFN- $\gamma$, TNF- $\alpha$ and IL-6, IL-17 was not produced by spleen cells from normal mice. The highest production of
IL-17 was observed in infections caused by the more arthritogenic strains, i.e., ATCC $19095 \mathrm{SEC}^{+}$and N315 ST5 TSST- $1^{+}$. The role of IL-17 as a mediator of joint destruction is being elucidated in experimental RA. The intra-articular injection of IL-17 into the knee results in joint inflammation and local damage [32]. This effect has been attributed, at least partially, to IL-17 induction of matrix metalloproteinases and also to its ability to promote osteoclastogenesis [34]. IL-17 also induces production of IL- 6 and IL- 8 by RA synovial fibroblasts via NF-kB and PI3-kinase/Akt-dependent pathways [35]. Furthermore, this cytokine induces production of chemokines and other pro-inflammatory cytokines such as TNF- $\alpha$, IL1 $\beta$, CXCL1 and CXCL5 [36,37]. These cytokines affect bone remodeling by stimulating proliferation and differentiation of osteoclast progenitors into mature osteoclasts $[38,39]$. Contrasting with this well-established role of IL17 in RA, the role of IL-17 in S. aureus-induced arthritis is not well understood. To our knowledge, this higher production of IL-17 in mice infected with the most arthritogenic strains is being described for the first time. Although this cytokine has been associated with protection in animals immunized with clumping factor A and also with local host defense during $S$. aureus-induced arthritis, its arthritogenic contribution in SA is still not disclosed. In this sense, these results support the possibility that higher IL-17 inducer S. aureus strains are endowed with stronger arthritogenic abilities $[26,40]$.

\section{Conclusions}

Altogether, these results demonstrated that $S$. aureus strains, isolated from biological samples, were able to induce typical septic arthritis in mice. These results also suggest that the variable arthritogenicity of these strains was, at least in part, related to their differential ability to induce IL-17 production.

\section{Abbreviations \\ ATCC: American type culture collection; BHI: Brain heart infusion; CFU: Colony forming units; ConA: Concanavalin A; NF-kB: Nuclear factor kappa B; RA: Rheumatoid arthritis; SA: Septic arthritis; SAg: Superantigen; SEC: Staphylococcal enterotoxin C; SEA: Staphylococcal enterotoxin A: TSST: Toxic shock syndrome toxin; CEEA: Ethcis committee for animal experimentation; LS-1: Paracoccus seriniphilus sp. nov.; ELISA: Enzyme linked immunosorbent assay; SE: Standard error; BD: Becton Dickinson and Company; R\&D: Research and development.}

\section{Competing interests}

The authors declare that they have no competing interests.

\section{Authors' contributions}

This study was conceived by PMCM and AS. All authors contributed to carry out the experiments, read and approved the final manuscript.

\section{Acknowledgements}

This study was supported by grant 2011/04323-3, São Paulo Research Foundation (FAPESP) and grant 472589/2011-3, Conselho Nacional de Desenvolvimento Científico e Tecnológico (CNPq). We are also grateful to the technical support of Daniele Santi Ceolin and Patricia de Sá Mortagua Germino. 


\section{Author details}

${ }^{1}$ Department of Microbiology and Immunology, Biosciences Institute, Univ. Estadual Paulista (UNESP), Distrito de Rubião Júnior s/n, 18618-070 Botucatu, São Paulo, Brazil. ${ }^{2}$ Department of Biological Sciences, School of Dentistry of Bauru, São Paulo University-FOB/USP, Bauru, São Paulo, Brazil.

Received: 1 May 2013 Accepted: 27 August 2013

Published: 30 August 2013

\section{References}

1. Edwards AM, Massey RC: How does Staphylococcus aureus escape the bloodstream? Trends Microbiology 2011, 4:184-190.

2. Tarkowski A: Infectious arthritis. Best Pract Res Clin Rheumatol 2006, 20:1029-1044.

3. Nade S: Septic arthritis. Best Pract Res Clin Rheumatol 2003, 17:183-200.

4. Bremell T, Abdelnour A, Tarkowski A: Histopathological and serological progression of experimental Staphylococcus aureus arthritis. Infect Immun 1992, 60:2976-2985

5. Verdrengh M, Tarkowski A: Role of macrophages in Staphylococcus aureusinduced arthritis and sepsis. Arthritis \& Rheumatism 2000, 43:2276-2282.

6. Saidenberg-Kermanac'h N, Corrado A, Lemeiter D, Devernejoul MC, Boissie MC, Cohen-Solal M: TNF-alpha antibodies and osteoprotegerin decrease systemic bone loss associated with inflammation through distinct mechanisms in collagen induced arthritis. Bone 2004, 35:1200-1207.

7. Satorres SE, Alcaráz LE, Cargnelutti E, Di Genaro MS: IFN-gamma plays a detrimental role in murine defense against nasal colonization of Staphylococcus aureus. Immunol Lett 2009, 2:185-188.

8. Braun T, Zwerina J: Positive regulators of osteoclastogenesis and bone resorption in rheumatoid arthritis. Arthritis Res Ther 2011, 4:222-235.

9. Kolls JK, Linden A: Interleukin-17 family members and inflammation. Immunity 2004, 21:467-476

10. Switalski LM, Patti JM, Butcher W, Gristina AG, Speziale P, Höök M: A collagen receptor on Staphylococcus aureus strains isolated from patients with septic arthritis mediates adhesion to cartilage. Mol Microbio/ 1993, 7:99-107.

11. Nilsson IM, Lee JC, Bremell T, Rydén C, Tarkowski A: The role of polysaccharide microcapsule expression in septicemia and septic arthritis. Infect Immun 1997, 65:4216-4221.

12. Josefsson E, Hartford O, O'Brien L, Patti JM, Foster T: Protection against experimental Staphylococcus aureus arthritis by vaccination with clumping factor A, a novel virulence determinant. J Infect Dis 2001, 184:1572-1580.

13. Pinchuk IV, Beswick EJ, Reyes VE: Staphylococcal enterotoxins. Toxins Basel 2010, 8:2177-2197.

14. Miethke T, Wahl C, Heeg K, Echtenacher B, Krammer PH, Wagner H: T cellmediated lethal shock triggered in mice by the superantigen staphylococcal enterotoxin B: critical role of tumor necrosis factor. J Exp Med 1992, 175:91-98.

15. Bremell T, Tarkowski A: Preferential induction of septic arthritis and mortality by superantigen-producing staphylococci. Infect Immun 1995, 63:4185-4187.

16. Tarkowski A, Vincent $C_{L}$, Gjertsson I, Hultgren $\mathrm{OH}$, Jonsson IM, Sakiniene $\mathrm{E}$ Verdrengh M: Model systems: modeling human staphylococcal arthritis and sepsis in the mouse. Trends Microbiol 2001, 9:21-326.

17. Bremell T, Lange S, Yacoub A, Rydén C, Tarkowski A: Experimental Staphylococcus aureus arthritis in mice. Infect Immun 1991, 59:2615-2623.

18. França TG, Ishikawa LL, Zorzella-Pezavento SF, Chiuso-Minicucci F, Guerino $C P$, Da Cunha ML, Sartori A: Immunization protected well-nourished mice but not undernourished ones from lung injury in methicillin-resistant Staphylococcus aureus (MRSA) infection. BMC Microbiol 2009, 9:240-247.

19. Bergdoll MS, Borja CR, Avena RM: Identification of a new enterotoxin as enterotoxin C. J Bacterio/ 1965, 90:1481-1485.

20. Hiramatsu K, Aritaka N, Hanaki H, Kawasaki S, Hosoda Y, Hori S, Fukuchi Y, Kobayashi I: Dissemination in Japanese hospitals of strains of Staphylococcus aureus heterogeneously resistant to vancomycin. Lancet 1997, 350:1670-1673.

21. Cunha MLRS, Calsolari AR, Araújo Júnior JP: Detection of enterotoxin and toxic shock syndrome toxin 1 genes in staphylococcus, with emphasis on coagulase-negative staphylococci. Microbiol Immunol 2007, 51:381-390.

22. De Boer ML, Chow AW: Toxic shock syndrome toxin 1-producing Staphylococcus aureus isolates contain the staphylococcal enterotoxin B genetic element but do not express staphylococcal enterotoxin B. J Infect Dis 1994, 4:818-827.

23. Casman EP: Designation of staphylococcal enterotoxins. J Bacterio/ 1963, 85:715-716.

24. Abdelnour A, Arvidson S, Bremell T, Rydén C, Tarkowski A: The accessory gene regulator (agr) controls Staphylococcus aureus virulence in a murine arthritis model. Infect Immun 1993, 61:3879-3885.

25. Jonsson IM, Arvidson S, Foster S, Tarkowski A: Sigma factor B and RsbU are required for virulence in Staphylococcus aureus-induced arthritis and sepsis. Infect Immun 2004, 72:6106-6111.

26. Henningsson $L$, Jirholt $P$, Lindholm $C$, Eneljung $T$, Silverpil $E$, Iwakura $Y$, Linden A, Gjertsson I: Interleukin-17A during local and systemic Staphylococcus aureus-induced arthritis in mice. Infect Immun 2010, 78:3783-3790

27. Ymele-leki P, Ross JM: Erosion from Staphylococcus aureus biofilms grown under physiologically relevant fluid shear forces yields bacterial cells with reduced avidity to collagen. Appl Environ Microbiol 2007, 73:1834-1841.

28. Brooks $J \mathrm{~L}$, Jefferson KK: Staphylococcal biofilms: quest for the magic bullet. Adv Appl Microbiol 2012, 81:63-87.

29. Morgan DS, Fisher D, Merianos A, Currie BJ: An 18 year clinical review of septic arthritis from tropical Australia. Epidemiol Infect 1996, 117:423-428

30. Goldenberg DL, Reed Jl: Bacterial arthritis. N Engl J Med 1985, 12:764-771.

31. Goldenberg DL: Septic arthritis. Lancet 1998, 9097:197-202.

32. Li X, Yuan FL, Lu WG, Zhao YQ, Li CW, Li JP, Xu RS: The role of interleukin17 in mediating joint destruction in rheumatoid arthritis. Biochem Biophys Res Commun 2010, 2:131-135.

33. Zhu F, Wang P, Lee MNH, Goldring B, Konstantopoulos K: Prolonged application of high fluid shear to chondrocytes recapitulates gene expression profiles associated with osteoarthritis. Plos One 2010, $12: 15174$.

34. Sarkar S, Cooney LA, Fox DA: The role of T helper type 17 cells in inflammatory arthritis. Clin Exp Immunol 2010, 3:225-237.

35. Hwang SY, Kim JY, Kim KW, Park MK, Moon Y, Kim WU, Kim H: IL-17 induces production of IL- 6 and IL-8 in rheumatoid arthritis synovial fibroblasts via NF-kappaB- and PI3-kinase/Akt-dependent pathways. Arthritis Res Ther 2004, 6:R120-R128.

36. Ruddy MJ, Shen F, Smith JB, Sharma A, Gaffen SL: Interleukin-17 regulates expression of the CXC chemokine LIX/CXCL5 in osteoblasts: implications for inflammation and neutrophil recruitment. J Leukoc Biol 2004, 76:135-144.

37. Onishi RM, Gaffen SL: Interleukin-17 and its target genes: mechanisms of interleukin-17 function in disease. Immunology 2010, 129:311-321.

38. Zou W, Hakim I, Tschoep K, Endres S, Bar-Shavit Z: Tumor necrosis factoralpha mediates RANK ligand stimulation of osteoclast differentiation by an autocrine mechanism. J Cell Biochem 2001, 83:70-83.

39. Wright JA, Nair SP: Interaction of staphylococci with bone. Int J Med Microbiol 2010, 2-3:193-204.

40. Narita K, Dong-Liang H, Mori F, Wakabayashi K, Iwakura Y, Nakane A: Role of interleukin-17A in cell-mediated protection against Staphylococcus aureus infection in mice immunized with the fibrinogen-binding domain of clumping factor A. Infect Immun 2010, 78:4234-4242.

doi:10.1186/1471-2334-13-400

Cite this article as: Colavite-Machado et al.: Differential arthritogenicity of Staphylococcus aureus strains isolated from biological samples. BMC Infectious Diseases 2013 13:400. 